\title{
Undifferentiated Uterine Sarcoma: A Rare Cause of Pelvic Pain in Young Woman
}

\author{
Fenohery Nalisoa Rakotondrainibe ${ }^{1 *}$, Zo Irène Raivoherivony ${ }^{2}$, \\ Nantenaina Soa Randrianjafisamindrakotroka ${ }^{2}$ \\ ${ }^{1}$ Pathological Anatomy Unit of Anosiala University Hospital, Antananarivo, Madagascar \\ ${ }^{2}$ Pathological Anatomy Unit of Joseph Ravoahangy Andrianavalona University Hospital, Antananarivo, Madagascar \\ Email: ${ }^{\star}$ fenoherynalisoa@gmail.com
}

How to cite this paper: Rakotondrainibe, F.N., Raivoherivony, Z.I. and Randrianjafisamindrakotroka, N.S. (2019) Undifferentiated Uterine Sarcoma: A Rare Cause of Pelvic Pain in Young Woman. Open Journal of Pathology, 9, 71-75.

https://doi.org/10.4236/ojpathology.2019.9 $\underline{4008}$

Received: June 20, 2019

Accepted: September 17, 2019

Published: September 20, 2019

Copyright $\odot 2019$ by author(s) and Scientific Research Publishing Inc. This work is licensed under the Creative Commons Attribution International License (CC BY 4.0).

http://creativecommons.org/licenses/by/4.0/

\begin{abstract}
We report a case of undifferentiated uterine sarcoma occurring in a 20-year-old woman presenting as a pelvic pain with a mass discovered at the physical examination. The tumor ruptured into the abdominal cavity. A total hysterectomy was performed. On the pathological examination, the uterine corpus presents a proliferation of markedly atypical spindle cells admixed with round cells and pleomorphic cells. The tumor deeply infiltrated the myometrium. The tumor was very necrotizing. There were more than 50 mitoses per 10 fields at high magnification. The isthmus was invaded by the tumor. The diagnosis of undifferentiated uterine sarcoma was confirmed by immunohistochemistry. The tumor shows negativity for the following markers: CKAE1/AE3, h-caldesmon, $\alpha$-SMA, progesterone receptors, desmin, CD 10, alpha foeto-protein, CD 3 and CD 20.
\end{abstract}

\section{Keywords}

Sarcoma, Endometrial Stroma, Uterine Sarcoma

\section{Introduction}

Endometrial stromal tumors in their well-differentiated form are tumors made of cells that resemble proliferative phase endometrial stromal cells. These tumors present numerous spiral arteries with concentric disposition of the cells around these vascular structures of regular size and regularly dispersed [1].

Endometrial stromal tumors are rare uterine tumors. They constitute about $0.2 \%$ of all uterus malignancies. They are more common among black women [1].

During a period of ten years from 2007 to 2016, we diagnosed 12 cases of en- 
dometrial stromal tumors including eight low-grade endometrial stromal tumors, two high-grade endometrial stromal tumors, and two undifferentiated uterine sarcomas. Undifferentiated uterine sarcoma rarely occurs in young women. This pathology mostly affects menopausal women.

\section{Clinical Case}

The confidentiality of the patient was strictly respected for this study. A 20-year-old woman with no particularity in her medical history presented with a pelvic pain. She was nulliparous. The physical examination showed a large pelvic median rounded mass, about $15 \mathrm{~cm}$ in diameter, sensitive and mobile. At vaginal touch, this mass filled the anterior cul de sac, pushing back the cervix which was inaccessible to the finger. The pregnancy test was negative. Pelvic and endovaginal ultrasound showed a heterogeneous uterine mass of $15 \times 10 \times 10 \mathrm{~cm}$. The tumor was well vascularized on Doppler ultrasound. The abdominal ultrasound and the chest X-ray were without abnormality. The tumor increased rapidly in six days and resulted in peritoneal syndrome. Emergency laparotomy showed a bulky mass of the uterus ruptured into the abdominal cavity. The uterine mass measured $15 \mathrm{~cm}$ long axis. A total hysterectomy with adnexal preservation and partial omentectomy were performed. On gross examination, the uterus weighed $1950 \mathrm{~g}$ and measured $21 \times 19 \times 15 \mathrm{~cm}$. There was a rupture of the anterior wall with outcome of a cerebral greyish mass, with extensive necrosis. On histological examination, the uterine corpus presented a proliferation of markedly atypical spindle cells admixed with round and pleomorphic cells (Figure 1). The tumor deeply infiltrated the myometrium. The tumor was very necrotizing. The mitotic count was more than 50 mitoses per 10 fields at high magnification. Focally, we observed images of lymphatic invasion. The isthmus was invaded by the tumor. The cervix was free of neoplasm. Histological examination concluded to suspicion of undifferentiated uterine sarcoma. Immunohistochemical studies showed negativity of polyvalent cytokeratin ( $\mathrm{AE} 1 / \mathrm{AE} 3$ ), h-caldesmon, $\alpha$-SMA (Figure 2), progesterone receptors (Figure 3), desmin, CD 10, alpha foeto-protein, CD 3 and CD 20. The patient died six months after the diagnostic.

\section{Discussion}

Undifferentiated uterine sarcoma is a rare tumor. It represents less than $1 \%$ of uterus malignancies and less than $10 \%$ of uterine sarcomas [2] [3].

It presents clinically with menometrorrhagia, a pelvic mass and pelvic pain [2]. In our case, the tumor presented as a painful pelvic mass evolving towards a peritoneal syndrome.

Endometrial stromal tumors mainly occur in women between 40 and 55 years [2], but rare cases have been reported in young women [4] [5] [6]. Our patient is very young at the time of diagnosis. The prognosis in this age group is usually poor because most of the time, the disease is already in an advanced stage [4] [5]. 


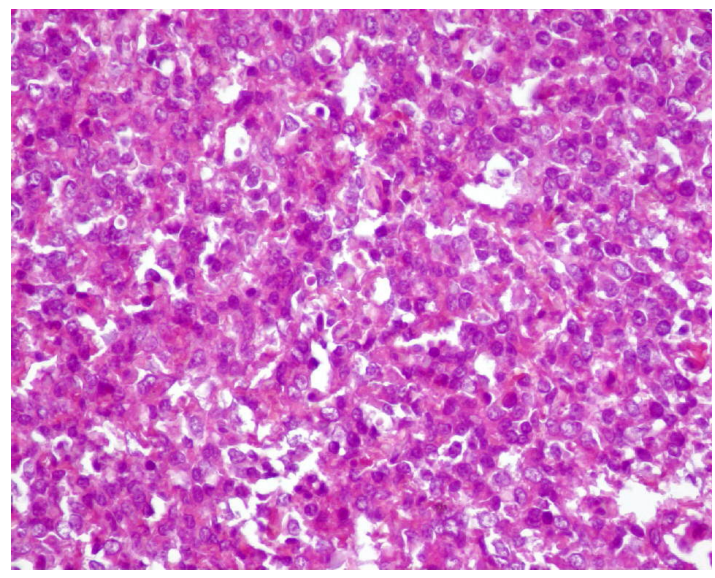

Figure 1. Total hysterectomy specimen. Undifferentiated uterine sarcoma. Proliferation of spindle cells admixed with round and pleomorphic cells. Hematoxylin Eosin $\times 400$. Pathological Anatomy Unit of Joseph Ravoahangy Andrianavalona University Hospital.

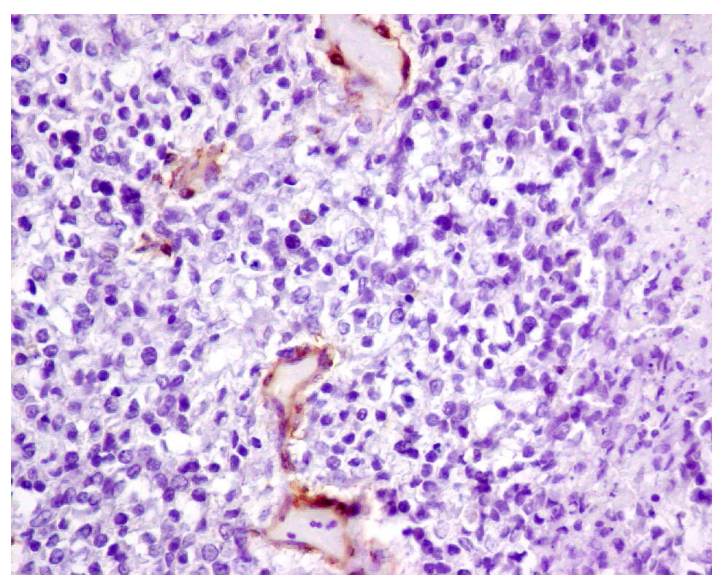

Figure 2. Total hysterectomy specimen. Undifferentiated uterine sarcoma. Undifferrenciated uterine sarcoma. $\alpha$-Smooth Muscle Actin negative immunolabeling with positive internal vessel control. Immunohistochemistry $\times$ 400. Pathological Anatomy Unit, Haut Levêque Bordeaux University Hospital.

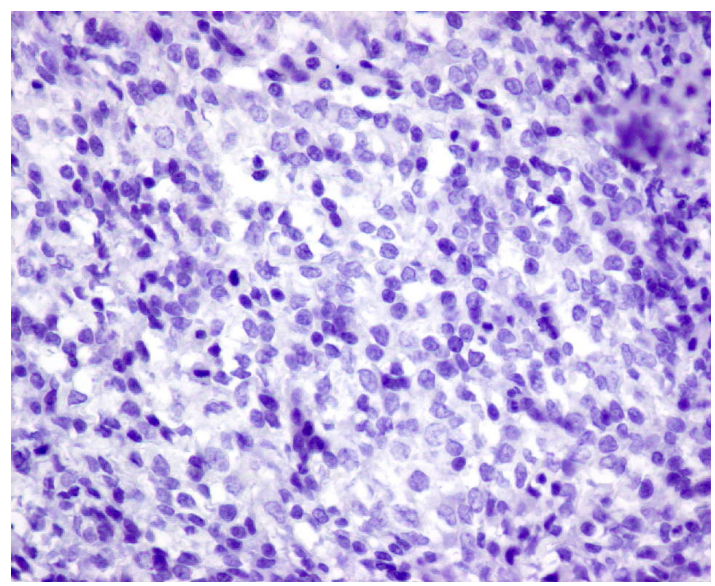

Figure 3. Total hysterectomy specimen. Undifferentiated uterine sarcoma. Negativity of progesteron receptors and presence of numerous mitosis. Immunohistochemistry $\times 400$. Pathological Anatomy Unit, Haut Levêque Bordeaux University Hospital. 
Morphologically, the WHO histopathological classification of uterine sarcomas was revised in 2003, 2009 and 2014. In the last classification, endometrial stromal tumors are divided into four main histological types: endometrial stromal nodule, low grade endometrial stromal sarcoma, high grade endometrial stromal sarcoma and undifferentiated uterine sarcoma [3]. In high-grade endometrial stromal sarcoma, there is a proliferation of atypical cells which morphology is not very similar to that of the endometrial stromal cells. The cells show marked cyto-nuclear atypias. Mitotic activity is high (more than 10 per 10 High Power Field). There are often abnormal mitosis [2] [3]. In our case, there were 50 mitoses in 10 consecutive fields at high magnification.

Endometrial stromal tumors should be distinguished from smooth muscle tumors, mesenchymal mixed malignant tumors and uterine metastasis of a gastro-intestinal stromal tumor (GIST). The immunohistochemical study is then of great utility for the diagnosis. CD 10 is the most sensitive marker of endometrial stromal tumors. However, its use in combination with other antibodies eliminates differential diagnoses [7] [8].

The desmin, h-caldesmon, CD10 and estrogen receptor panel is used to make the differential diagnosis with leiomyosarcoma. Carcinosarcoma and adenosarcoma are also differential diagnoses that must be eliminated prior to the diagnosis of endometrial stromal tumor. In our case, CD 10 was negative. The negativity of polyvalent cytokeratin (AE $1 / \mathrm{AE} 3$ and $\mathrm{CKL} 1$ ) is not in favour of the diagnosis of undifferentiated carcinoma. The negativity of caldesmone, $\alpha$-SMA and desmin is not in favour of the diagnosis of leiomyosarcoma. Lymphoid markers such as anti-CD3, anti-L26 and anti-CD20 antibodies were performed systematically and were also negative.

At the end of this immunohistochemical analysis, the diagnosis of undifferentiated uterine sarcoma was retained, since the tumor showed no differentiation.

Recently cotzia $\mathrm{P}$ et al. reported described new molecular alterations, such as YWHAE-NUTM2 fusions and BCOR genetic abnormalities in undifferentiated uterine sarcoma. Molecular studies were not performed for our case in absence of technical platform [9].

\section{Conclusion}

Undifferentiated uterine sarcoma is a tumor that affects pre-menopausal women and exceptionally young women. For our patient, the tumor was revealed by a painful pelvic mass evolving towards a peritoneal syndrome. Our case is distinguished by the rapid evolution of the disease. The lack of differentiation within the tumor, the presence of necrosis and the mitotic count are in favour of undifferentiated uterine sarcoma.

\section{Acknowledgements}

Thanks to the Pathological Anatomy unit of Haut Levêque Bordeaux Hospital for the immunohistochemistry studies for our case. 


\section{Conflicts of Interest}

The authors declare no conflicts of interest regarding the publication of this paper.

\section{References}

[1] Hendrickson, M.R., Tavassoli, F.A., Kempson, R.L., McCluggage, W.G., Haller, U. and Kubik-Huch, R.A. (2003) Mesenchymal Tumors and Related Lesions. In: Tavassoli, F.A. and Devilee, P., Eds., World Health Organization Classification of Tumors Pathology and Genetics of Tumors of the Breast and Female Genital Organs, IARC Press, Lyon, 233-244.

[2] Rauh-Hain, J.A. and del Carmen, M.G. (2013) Endometrial Stromal Sarcoma: A Systematic Review. Obstetrics \& Gynecology, 122, 676-683. https://doi.org/10.1097/AOG.0b013e3182a189ac

[3] Conklin, C.M.J. and Longacre, T.A. (2014) Endometrial Stromal Tumors: The new WHO Classification. Advances in Anatomic Pathology, 21, 383-393. https://doi.org/10.1097/PAP.0000000000000046

[4] Michalas, S., Creatsas, O., Deligeoroglou, E. and Markaki, S. (1994) High-Grade Endometrial Stromal Sarcoma in a 16-Year-Old Girl. Gynecologic Oncology, 54, 95-98. https://doi.org/10.1006/gyno.1994.1173

[5] Wang, K.-C., Liang, D.-C., Su, T.-H., Hung, F.-Y. and Yang, Y.-C. (1998) High-Grade Endometrial Stromal Sarcoma in a 10-Year-Old Girl: Case Report. Chang Gung Medical Journal, 21, 312.

[6] Emmert, C., Dietrich, F., Wecke, W.D. and Riedel, H.-H. (1999) High-Grade Endometrial Stromal Sarcoma (HG-ESS) in an Adolescent Girl with Benign Mediastinal Schwannoma. Journal of Obstetrics \& Gynaecology, 19, 210-212. https://doi.org/10.1080/01443619965697

[7] Zhu, X.-Q., Shi, Y.-F., Cheng, X.-D, Zhao, C.-L. and Wu, Y.-Z. (2004) Immunohistochemical Markers in Differential Diagnosis of Endometrial Stromal Sarcoma and Cellular Leomyoma. Gynecologic Oncology, 92, 71-79. https://doi.org/10.1016/j.ygyno.2003.08.038

[8] Chu, P.G., Arber, D.A., Weiss, L.M. and Chang, K.L. (2001) Utility of CD10 in Distinguishing between Endometrial Stromal Sarcoma and Uterine Smooth Muscle Tumors: An Immunohistochemical Comparison of 34 Cases. Modern Pathology, 14, 465-471. https://doi.org/10.1038/modpathol.3880335

[9] Cotzia, P., Benayed, R., Mullaney, K., Oliva, E., Felix, A., Ferreira, J., et al. (2019) Undifferentiated Uterine Sarcomas Represent Under-Recognized High-Grade Endometrial Stromal Sarcomas. The American Journal of Surgical Pathology, 43, 662-669. https://doi.org/10.1097/PAS.0000000000001215 